\title{
Entropy of Scalar Field in 3+1 Dimensional Constant Curvature Black Hole Background
}

\author{
K. Ghosh円 \\ Saha Institute of Nuclear Physics, 1/AF, Bidhannagar, Calcutta - 700064, India.
}

\begin{abstract}
We consider the thermodynamics of minimally coupled massive scalar field in $3+1$ dimensional constant curvature black hole background. The brick wall model of 't Hooft is used. When Scharzschild like coordinates are used it is found that apart from the usual radial brick wall cut-off parammeter an angular cut-off parameter is required to regularize the solution. Free energy of the scalar field is obtained through counting of states using the WKB approximation. It is found that the free energy and the entropy are logarithmically divergent in both the cut-off parameters.
\end{abstract}




\section{Introduction}

A black hole has a horizon beyond which no matter or information can escape. The absence of information about the region inside the horizon manifests itself in an entropy. A quantitative expression for the entropy and the laws of black hole thermodynamics were first obtained by Bekenstein [1] mainly on the basis of analogy. Since then a lot of effort has been devoted to explain this entropy on a statistical mechanics basis. A related issue is the entropy of quantum fields in black hole backgrounds. The entropy of quantum fields is obtained by various methods, e.g., by tracing over local degrees of freedom inside the horizon (geometric entropy) [2], by explicit counting of degrees of freedom of the fields propagating outside the horizon (entanglement entropy) [3,4,5] or by the Euclidean path integral $[6,7]$. These expressions are proportional to the area of the horizon and constitute the first quantum correction to the gravitational entropy. Divergences appear in the density of the states associated to the horizon and can be absorbed in the renormalized expression of the gravitational coupling constant [8]. To regularize these divergences 't Hooft proposed [3] that the field modes should be cut off in the vicinity of the horizon by imposing a brick wall cut-off. This method has been used to study the entropy of matter around different black hole solutions.

A new type of black hole solution has been found by Bañados [9]. This solution results from an identification of space-time points in anti-de Sitter space and represents a higher dimensional generalization of the $2+1$ dimensional BTZ black hole [10] These are constant curvature black holes $(\mathrm{CCBH})$ with a negative cosmological constant. However the spatial topology is toroidal. Mann and Creighton considered the thermodynamics of the $3+1$ dimensional constant curvature black holes as a solution to the equations of general relativity with a negative cosmological constant [11]. They used a quasilocal foliation which is degenerate along a particular direction. The entropy is obtained from the microcanonical action evaluated in the Euclidean sector of the black hole space-time. The entropy is obtained as the integral of a Noether charge 2-form on the history of the boundary of the quasilocal surface. The entropy is unusual in the sence that it differs from the usual expression of a quarter of the horizon surface area. Moreover, the entropy vanishes as the boundary of the quasilocal suface used to foliate the space-time is pulled back to the horizon. Thus the entropy seems to be associated with the surface of degeneracy of the foliation used rather than the horizon surface area.

In this context it is natural to enquire about the entropy of quantum fields defined on such backgrounds. Thus we investigate the thermodynamical behaviour of a real scalar field propagating on the $3+1$ dimensional constant curvature black hole using the brick wall cut-off.

\section{Wave equation for scalar fields in $3+1$ dimensional CCBH black holes:}

The anti-de Sitter space in $3+1$ dimension is defined as the universal covering space of the hypersurface

$$
-x_{0}^{2}+x_{1}^{2}+x_{2}^{2}+x_{3}^{2}-x_{4}^{2}=-l^{2} .
$$


metric for $3+1$ dimensional $\mathrm{CCBH}$ is given by [9]

$$
d s^{2}=\frac{l^{4} f^{2}(r)}{r_{h}^{2}}\left[d \theta^{2}-\cos ^{2} \theta(d t / l)^{2}\right]+\frac{d r^{2}}{f^{2}(r)}+r^{2} d \phi^{2}
$$

where $f(r)=\left(\frac{r^{2}-r_{h}^{2}}{l^{2}}\right)$. These coordinates are valid outside the horizon $\left(r>r_{h}\right)$ for $0 \leq \theta<2 \pi$ and $0 \leq \phi<2 \pi$. However in these coordinates only part of the space is covered. It is clear that the foliation becomes degenerate along the direction $\theta=\pi / 2$ and $\theta=3 \pi / 2$.

The wave equation for a minimally coupled scalar field in a curved background is

$$
\frac{1}{\sqrt{-g}} \partial_{\mu}\left(\sqrt{-g} g^{\mu \nu} \partial_{\nu} \psi\right)-m^{2} \psi=0
$$

In this case it leads to

$$
\begin{aligned}
-\frac{l^{2}}{\cos ^{2} \theta} \partial_{t}^{2} \psi & +\frac{1}{\cos \theta} \partial_{\theta}\left(\cos \theta \partial_{\theta} \psi\right)+\frac{l^{4} f(r)}{r r_{h}^{2}} \partial_{r}\left[r f^{3}(r) \partial_{r} \psi\right] \\
& +\frac{l^{4} f^{2}(r)}{r^{2} r_{h}^{2}} \partial_{\phi}^{2} \psi-\frac{m^{2} l^{4} f^{2}(r)}{r_{h}^{2}} \psi=0
\end{aligned}
$$

Since there is no explicit time dependence in the foliation used, we may construct stationary state solutions. We take the stationary state solutions to be of the form

$$
\psi=K e^{i E t} e^{i N \phi} P(\theta) R(r)
$$

where $N$ is an integer, $K$ a normalization constant, $E$ a real parameter. We have, using the separation of variables,

$$
\frac{1}{\cos \theta} \partial_{\theta}\left(\cos \theta \partial_{\theta} P\right)+\left[\nu(\nu+1)+\frac{E^{2} l^{2}}{\cos ^{2} \theta}\right] P=0
$$

where $\nu$ is an arbitrary complex parameter. For the radial part, we have,

$$
\partial_{r}\left[r f^{3}(r) \partial_{r} R\right]-\left[\frac{N^{2} f(r)}{r}+m^{2} r f(r)+\frac{\nu(\nu+1) r r_{h}^{2}}{l^{4} f(r)}\right] R=0 .
$$

We want to calculate the entropy of the scalar field. For this purpose we use the WKB approximation to the radial differential equation to obtain the radial degeneracy factor associated with the brick wall boundary condition. Wehave to find out the quantization condition on the parameters of the angular equation using appropriate boundary conditions.

\section{Calculation of entropy}

The equation for the radial part is given by

$$
\left(r^{2}-r_{h}^{2}\right) \partial_{r}^{2} R+\left[4 r-\frac{r_{h}^{2}}{r}\right] \partial_{r} R-\left[\frac{N^{2} l^{2}}{r^{2}}+m^{2} l^{2}+\frac{\nu(\nu+1) r_{h}^{2}}{\left(r^{2}-r_{h}^{2}\right)}\right] R=0
$$


space-time in which the $\mathrm{CCBH}$ is immersed is not globally hyperbolic. The infinity in this case is timelike. Fresh information and matter can come in or leak out through this time-like infinity in finite coordinate time. Moreover any initial data of compact support on a Cauchy surface will not remain so on other space-like surfaces. The authors of [12] have considered in detail the quantization of scalar fields in adS and its covering spaces. A consistent scheme can be devised if we take the following boundary conditions:

(1) Dirichlet boundary condition:

$$
\lim _{r \rightarrow \infty} \sqrt{r} R=0
$$

(2) Newmann boundary condition:

$$
\lim _{r \rightarrow \infty} r^{\frac{3}{2}} \frac{d}{d r}\left(\frac{1}{\sqrt{r}} R\right)=0
$$

The solution of equ.(16) for large $r$ is given by

$$
R=\frac{1}{\sqrt{r^{3}}}\left[C_{1} r^{\sqrt{\frac{\left(9+4 m^{2} l^{2}\right)}{2}}}+C_{2} r^{-\sqrt{\frac{\left(9+4 m^{2} l^{2}\right)}{2}}}\right] .
$$

For real particles $\left(m^{2}=0\right)$, only the second part of the solution is acceptable since it is in accordance with the boundary condition at infinity.

However we are interested in the degeneracy of the field modes near the horizon. We use the WKB approximation, i.e, we write $R(r)=\rho(r) e^{i S(r)}$, where $\rho(r)$ is a slowly varying amplitude and $S(r)$ is a rapidly varying phase factor. When substituted in equ.(8), this gives us the following value for the $r$-dependent wave number

$$
k^{2}=-\left[\frac{\nu(\nu+1) r_{h}^{2}}{\left(r^{2}-r_{h}^{2}\right)^{2}}+\frac{m^{2} l^{2}}{\left(r^{2}-r_{h}^{2}\right)}+\frac{N^{2} l^{2}}{r^{2}\left(r^{2}-r_{h}^{2}\right)}\right]
$$

We will consider only those values of $\nu$ for which $k(r)$ is real. In the near horizon region there are two sectors of the values of $\nu$ for which it is possible, viz.,

$$
\nu=-\frac{1}{2}+i \chi
$$

where $\chi$ is a real parameter, and

$$
\nu=-\lambda(1-\lambda)
$$

where $\lambda$ is a real parameter with the range $0 \leq \lambda \leq 1$.

The values of $\nu$ are further restricted by the following semiclassical quantization condition,

$$
n_{r} \pi=\int d r k(r, \nu, N)
$$

where $n_{r}$ is a nonnegative integer. This gives us the radial degeneracy of the mode functions.

For the first sector, we have,

$$
n_{r}(\chi, N)=\frac{1}{2} \int^{L_{2}} d r\left[\frac{\left(\chi^{2}+\frac{1}{4}\right) r_{h}^{2}}{\left(m^{2} 2\right)^{2}}-\frac{N^{2} l^{2}}{2\left(m^{2}\right.}-\frac{m^{2} l^{2}}{\left(m^{2}\right)}\right]^{\frac{1}{2}}
$$


and for the second sector we have,

$$
n_{r}(\lambda, N)=\frac{1}{\pi} \int_{r_{h}+\epsilon}^{L_{1}} d r\left[\frac{\lambda(1-\lambda) r^{2}{ }_{h}}{\left(r^{2}-r_{h}^{2}\right)^{2}}-\frac{N^{2} l^{2}}{r^{2}\left(r^{2}-r_{h}^{2}\right)}-\frac{m^{2} l^{2}}{\left(r^{2}-r_{h}^{2}\right)}\right]^{\frac{1}{2}}
$$

In the above two expressions we cannot take the upper limits of integration, i.e, $L_{1}$ and $L_{2}$ to be arbitrarily large. Then the mass term will become large and the solution will no longer remain oscillatory. This is expected from the point of view of the discussion given below equ.(8).

To solve equation (6) and obtain quantization condition on $E$, we put $\mu= \pm i E l$. Then equ. (6) becomes

$$
\frac{1}{\cos \theta} \partial_{\theta}\left(\cos \theta \partial_{\theta} P\right)+\left[\nu(\nu+1)-\frac{\mu^{2}}{\cos ^{2} \theta}\right] P=0 .
$$

The solution of this equation is $P_{\nu}^{ \pm \mu}( \pm x)$, where $x=\sin \theta$, and

$$
P_{\nu}^{\mu}(x)=\frac{1}{\Gamma(1-\mu)}\left(\frac{1+x}{1-x}\right)^{\frac{\mu}{2}} F\left(-\nu, \nu+1 ; 1-\mu ; \frac{1-x}{2}\right)
$$

with the condition that $(\nu+\mu) \neq$ an integer. Here $F\left(-\nu, \nu+1 ; 1-\mu ; \frac{1-x}{2}\right)$ is the hypergeometric function [13]. However this solution is divergent at $x= \pm 1$, i.e, along $\theta=\frac{\pi}{2}, \frac{3 \pi}{2}$, which is expected because the foliation used is degenerate in those directions.

A consistent regularization scheme, which makes the solution vanish at $x= \pm(1 \mp \eta)$ and thus avoids the problem associated with the degeneracy of the foliation used, can be devised if we chose the solution to be of the following form:

$$
u=C_{1} P_{\nu}^{i E l}(x)+C_{2} P_{\bar{\nu}}^{-i E l}(x)
$$

for $0 \leq x$, i.e, for $0 \leq \theta<\pi$ and

$$
u=C_{3} P_{\nu}^{i E l}(-x)+C_{4} P_{\bar{\nu}}^{-i E l}(-x)
$$

for $0 \geq x$, i.e, for $\pi \leq \theta<2 \pi$.

The constants $C_{1}, C_{2}, C_{3}$ and $C_{4}$ should be chosen in such a way that the solutions are real. Moreover the two forms of the solutions in the two regions and their first derivatives should match along $\theta=0$ and $\theta=\pi$. However, for the first sector of the given values of $\nu$ as determined from equ.(11), the solution (17) is of the form of conical function [13]. The solution will satisfy all the conditions as stated above for the following values of [14] $C_{1}, C_{2}, C_{3}$ and $C_{4}$.

$$
C_{1}=-C_{3}=\frac{i \Gamma\left(\frac{3}{4}-\frac{i \chi}{2}-\frac{i E l}{2}\right) \Gamma\left(\frac{3}{4}+\frac{i \chi}{2}-\frac{i E l}{2}\right)}{2^{i E l} \sqrt{\pi}}
$$

and

$$
C_{2}=-C_{4}=\frac{-i \Gamma\left(\frac{3}{4}-\frac{i \chi}{2}+\frac{i E l}{2}\right) \Gamma\left(\frac{3}{4}+\frac{i \chi}{2}+\frac{i E l}{2}\right)}{2^{-i E l} \sqrt{\pi}}
$$

Demanding $u=0$ for $x= \pm(1 \mp \eta)$, gives us,

$$
\Gamma\left(\frac{3}{4}-\frac{i \chi}{2}-\frac{i E l}{2}\right) \Gamma\left(\frac{3}{4}+\frac{i \chi}{2}-\frac{i E l}{2}\right), 1_{,} \frac{i E l}{2}
$$


where c.c denotes the complex conjugate of the first term. Here the two gamma funtions are complex conjugate to each other and the phase factor involves $\chi$ and $E$. With proper choice of the normalization constant, we have,

$$
e^{i\left[\frac{E l}{2} \ln \left(\frac{1}{2 \eta}\right)+\pi \alpha_{1}(E, \chi)\right]}-c . c=0 .
$$

Here $\alpha_{1}$ is a function of $E$ and $\chi$ such that $0 \leq\left|\alpha_{1}\right|<1$. Hence we have, from equ.(20),

$$
\frac{E l}{2} \ln \left(\frac{1}{2 \eta}\right)+\pi \alpha_{1}=n_{1} \pi
$$

where $n_{1}$ is an integer. Now in the above relation as $\eta \rightarrow 0$, both the r.h.s, and the first term in the l.h.s become very large compared to the second term in the l.h.s. Hence we have

$$
E=\frac{2 n_{1} \pi}{l\left[\ln \left(\frac{1}{2 \eta}\right)\right]}
$$

Clearly the solution diverges as $\eta \rightarrow 0$.

Proceeding in the same way for the second sector (equ.(12)) of $\nu$ we obtain another set of values of $\mathrm{E}$ :

$$
\frac{\Gamma\left(1-\frac{\lambda}{2}-\frac{i E l}{2}\right) \Gamma\left(\frac{1}{2}+\frac{\lambda}{2}-\frac{i E l}{2}\right)}{\Gamma(1-i E l)}\left(\frac{1}{2 \eta}\right)^{\frac{i E l}{2}}-c . c=0
$$

This condition gives us another set of values of $\mathrm{E}$ :

$$
\frac{E l}{2}\left[\ln \left(\frac{1}{2 \eta}\right)\right]+\pi \alpha_{2}(E, \lambda)=n_{2} \pi
$$

where $n_{2}$ is an integer and $0 \leq\left|\alpha_{2}\right|<1$. Now proceeding in the same way as the first case we have,

$$
E=\frac{2 n_{2} \pi}{l\left[\ln \left(\frac{1}{2 \eta}\right)\right]}
$$

Clearly this solution also diverges for $\eta \rightarrow 0$.

The free energy of the scalar field is given by

$$
\beta F=\sum_{\text {degeneracies }} \sum_{n} \ln \left(1-e^{-\beta E_{n}}\right)
$$

In this case we have two sectors of $\nu$ and correspondingly, two sets of values of the energy levels. Hence the free energy of the scalar field is given by the following expression,

$$
\begin{aligned}
\beta F= & \frac{1}{\pi} \sum_{N} \int_{0}^{1} d \lambda \int_{r_{h}+\epsilon}^{L_{1}} d r k(r, \lambda, N) \sum_{n_{1}} \ln \left(1-e^{-\beta E_{n_{1}}}\right) \\
& +\frac{1}{\pi} \sum_{N} \int_{-\chi_{1}}^{\chi_{1}} d \chi \int_{r_{h}+\epsilon}^{L_{2}} d r k(r, \chi, N) \sum_{n_{2}} \ln \left(1-e^{-\beta E_{n_{2}}}\right)
\end{aligned}
$$

Here $\epsilon$ is the radial brick wall cut-off parameter. It is evident from equ.(14) and equ.(15) that the respective expressions for $k(r, \chi, N)$ and $k(r, \lambda, N)$ are divergent as the brick wall is 
For the degeneracy factors associated with energy, we have from equ. (23), and equ. (24)

$$
d E=\frac{2 \pi}{l} \frac{d n}{\ln \left(\frac{1}{2 \eta}\right)}
$$

For the radial degeneracy factors we have from equ.(14) and equ.(15):

$$
\begin{aligned}
& g\left(\epsilon, L_{1}, \lambda, N\right)= \\
& \frac{1}{2 \pi r_{h}^{2}}\left[\sqrt{\lambda(1-\lambda) r^{2} r_{h}^{2}-N^{2} l^{2}\left(r^{2}-r_{h}^{2}\right)-m^{2} l^{2} r^{2}\left(r^{2}-r_{h}^{2}\right)} \ln \left(\frac{r^{2}-r_{h}{ }^{2}}{r^{2}}\right)\right]_{r_{h}}^{L_{1}}- \\
& \frac{1}{4 \pi r_{h}{ }^{2}} \int_{r_{h}+\epsilon}^{L_{1}} \ln \left(\frac{r^{2}-r_{h}{ }^{2}}{r^{2}}\right) \frac{2 \lambda(1-\lambda) r r_{h}{ }^{2}-2 N^{2} l^{2} r-4 m^{2} l^{2} r^{3}+2 m^{2} l^{2} r r_{h}{ }^{2}}{\sqrt{\lambda(1-\lambda) r^{2} r_{h}{ }^{2}-N^{2} l^{2}\left(r^{2}-r_{h}{ }^{2}\right)-m^{2} l^{2} r^{2}\left(r^{2}-r_{h}^{2}\right)}} d r
\end{aligned}
$$

and a similar expression for $g\left(\epsilon, L_{2}, \chi, N\right)$ with $\lambda(1-\lambda)$ replaced by $\left(\chi^{2}+\frac{1}{4}\right)$ and $L_{1}$ replaced by $L_{2}$ in the above equation. From these expressions we have the following expression for the free energy of the scalar field,

$$
\begin{aligned}
\beta F= & \frac{l}{2 \pi} \sum_{N}\left[\int_{0}^{1} d \lambda g\left(\epsilon, L_{1}, \lambda, N\right)\right]\left[\ln \left(\frac{1}{2 \eta}\right) \int d E \ln \left(1-e^{-\beta E}\right)\right] \\
& +\frac{l}{2 \pi} \sum_{N}\left[\int_{0}^{1} d \lambda g\left(\epsilon, L_{2}, \chi, N\right)\right]\left[\ln \left(\frac{1}{2 \eta}\right) \int d E \ln \left(1-e^{-\beta E}\right)\right]
\end{aligned}
$$

Now we are interested in the divergence of the field modes near the horizon, i.e, the divergence appearing for small values of $\epsilon$. This gives the usual ultraviolet divergence of the free energy of the scalar field similar the case of Schwarzschild or Reissner-Nordstrom [3,5] black holes. Hence confining our attention to the near horizon region we have the following expression for the free energy:

$$
\begin{aligned}
\beta F= & \frac{l}{2 \pi} \sum_{N}\left[\int_{0}^{1} d \lambda g(\epsilon, \lambda, N)\right]\left[\ln \left(\frac{1}{2 \eta}\right) \int d E \ln \left(1-e^{-\beta E}\right)\right] \\
& +\frac{l}{2 \pi} \sum_{N}\left[\int_{0}^{1} d \lambda g(\epsilon, \chi, N)\right]\left[\ln \left(\frac{1}{2 \eta}\right) \int d E \ln \left(1-e^{-\beta E}\right)\right]
\end{aligned}
$$

where $g(\epsilon, \lambda, N)$ and $g(\epsilon, \chi, N)$, the ultraviolet divergent parts of the radial degeneracy factors are given by:

$$
g(\epsilon, \lambda, N)=-\frac{1}{2 \pi r_{h}^{2}} \ln \left(\frac{2 \epsilon}{r_{h}}\right)\left[r_{h}^{4} \lambda(1-\lambda)-2 \epsilon r_{h} N^{2} l^{2}-2 \epsilon r_{h}^{3} m^{2} l^{2}\right]^{\frac{1}{2}}
$$

and

$$
g(\epsilon, \chi, N)=-\frac{1}{2 \pi r_{h}^{2}} \ln \left(\frac{2 \epsilon}{r_{h}}\right)\left[r_{h}^{4}\left(\chi^{2}+\frac{1}{4}\right)-2 \epsilon r_{h} N^{2} l^{2}-2 \epsilon r_{h}^{3} m^{2} l^{2}\right]^{\frac{1}{2}}
$$

Hence to the leading order in $\epsilon$ we have the following expression for the free energy of the scalar field:

$$
N_{1} l
$$


where the $N$ integration is cut-off at some large but finite values $N_{1}$. The free energy is clearly divergent as the brick wall is pulled back on the horizon with an additional logarithmic divergence in the $\theta$ - cut-off $\eta$.

The entropy is given by

$$
S=\beta^{2} \frac{d F}{d \beta}
$$

This gives

$$
S=\frac{N_{1} l}{16 \pi \beta}\left[\pi+4 \chi_{1} \sqrt{4 \chi_{1}^{2}+1}+4 \ln \left(\sqrt{4 \chi_{1}^{2}+1}-2 \chi\right)\right]\left[\ln \left(\frac{1}{2 \eta}\right)\right]\left[\ln \left(\frac{r_{h}}{2 \epsilon}\right)\right]
$$

Now we take the inverse temperature to be the period of the time coordinate in the Euclidean sector of the metric to avoid the conical singularity. In this case it is $2 \pi l$. Substituting in equ.(36), we have

$$
S=\frac{N_{1}}{32 \pi^{2}}\left[\pi+4 \chi_{1} \sqrt{4 \chi_{1}^{2}+1}+4 \ln \left(\sqrt{4 \chi_{1}^{2}+1}-2 \chi\right)\right]\left[\ln \left(\frac{1}{2 \eta}\right)\right]\left[\ln \left(\frac{r_{h}}{2 \epsilon}\right)\right]
$$

Thus the entropy is not proportional to the horizon surface area which, in this case, is given by $2 \pi r_{h}$. However, it is logarithmically divergent in both the radial coordinate $r$ and angular coordinate $\theta$.

\section{Discussion}

In conclusion, we have considered the thermodynamics of a scalar field in $3+1$-dimensional $\mathrm{CCBH}$ background. In order to regulate the solution of the scalar field wave equation we have introduced two cut-off parameters. The first one is the usual radial (brick wall) cutoff parameter $\epsilon$ which regulates the solution near the horizon and the second one is the cut-off parameter $\eta$ in the angular coordinate $\theta$. We have calculated the entropy using the WKB approximation and found it to be logarithmically divergent in both of these cut-off parameters.

The expression for entropy is remarkable. Firstly, it is not proportional to the area $\left(2 \pi r_{h}\right)$ of the horizon. However, this behaviour is also reflected in the expression for the gravitational entropy of the $3+1$-dimensional $\mathrm{CCBH}$ which is given by [11]:

$$
S_{\text {grav }}=\pi l^{2} f(R)
$$

where $R$ is the radius of the quasilocal surface used to foliate the space-time. The gravitational entropy of $4+1$-dimensional rotating $\mathrm{CCBH}$ is also not proportional to the horizon surface area. So it seems to be a general feature associated with constant curvature black holes.

Secondly, the divergence of the scalar field entropy is closely related to the boundary conditions. The first, expected, diverging term, $\ln \left(\frac{\epsilon}{r_{h}}\right)$, is related to the brick wall boundary condition near the horizon. However the second diverging factor $\ln \left(\frac{2}{\eta}\right)$ is surprising because it is not seen in scalar field entropy in conventional black hole backgrounds (Schwarzschild or Reissner - Nordstrom). The reason for this factor is exactly the same as that which is 
metric has the same form as the $3+1$-dimensional $\mathrm{CCBH}$; the only difference is that the surfaces of constant $r$ and $t$ have the topology $S_{2} \otimes S_{1}$. It is expected that to regularize the solution of the scalar field wave equation a similar angular cut-off parameter will be necessary.

It may be noted that the study of scalar fields in BTZ black hole background $[15,16]$ has shown that the entropy of scalar fields depends on the method of calculation used. In this context it will be interesting to calculate the scalar field entropy in $3+1$-dimensional $\mathrm{CCBH}$ background using other techniques (e.g, heat kernal expansion or Hartle - Hawking Green function).

\section{Acknowledgments}

It is a great pleasure to the author to thank Prof. P. Mitra for many helpful discussions.

\section{References}

[1] J. Bekenstein, Phys. Rev. D 7, 2333 (1973)

[2] C. Callan and F. Wilczeck, Phys. Lett. B 333, 55 (1994)

[3] G. 't Hooft, Nucl. Phys. B 256, 727 (1985)

[4] R. B. Mann, L.Tarasov, and A.Zelnikov, Class. Quantum Grav. 9, 1487 (1992)

[5] A.Ghosh, and P.Mitra, Phys. Lett. B 357, 295 (1995)

[6] G. W. Gibbons, and S. W. Hawking, Phys. Rev. D 15, 2752 (1977)

[7] S. N. Solodukhin, Phys. Rev. D 51, 609 (1995)

[8] L. Susskind, and J. Uglum, Phys. Rev. D 50, 2700 (1994)

[9] M. Bañados, Phys. Rev. D 57, 1068 (1998)

[10] M. Bañados, M. Henneaux, C. Teiltelboim and J. Zanelli, Phys. Rev. D 48, 1506 (1993)

[11] J. D. E. Creighton and R. B. Mann, Phys. Rev. D 58, 024013, (1998)

[12] S. J. Avis, C. J. Isham and D. Storey, Phys. Rev. D 18, 3565 (1978)

[13] I. S. Gradshteyn and I. M. Ryzhik, Tables of Integrals Series and Products. Academic Press. (1965)

[14] M. Abramowitz, and A. Stegun, Handbook of Mathematical Functions. Dover Publications, Inc., New York. (1965)

[15] I. Ichinose and Y. Satoh, Nucl. Phys. B 447, 34 (1995)

[16] G. Lifschytz and M. Ortiz, Phys. Rev. D 49, 1929 (1994) 